\title{
Higher Frequency of Reoperation With a New Bicruciate-retaining Total Knee Arthroplasty
}

\author{
Jesse C. Christensen PT, DPT, Justin Brothers MD, Gregory J. Stoddard MPH, MBA, \\ Mike B. Anderson MSc, Christopher E. Pelt MD, Jeremy M. Gililland MD, \\ Christopher L. Peters MD
}

Published online: 4 April 2016

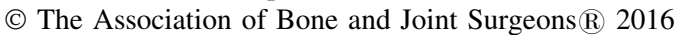

\begin{abstract}
Background With as many as $25 \%$ of patients reporting residual knee symptoms after primary total knee arthroplasty (TKA), alternative implant designs and surgical techniques have been proposed to further reduce these symptoms. There is growing evidence that retention of the anterior cruciate ligament (ACL) results in more natural knee kinematics; thus, implants with more normal joint
\end{abstract}

One of the authors certifies that he (CLP), or a member of his immediate family, has or may receive payments or benefits, during the study period, an amount of USD 100,001 to USD 1,000,000 from Biomet, Inc (Warsaw, IN, USA). One of the authors certifies that he (CEP), or a member of his immediate family, has or may receive payments or benefits, during the study period, an amount of USD 10,000 to USD 100,000 from Biomet, Inc. One of the authors certifies that he (JMG), or a member of his immediate family, has or may receive institutional research support, during the study period, an amount of USD 100,001 to USD \$1,000,000 from Biomet, Inc. All ICMJE Conflict of Interest Forms for authors and Clinical Orthopaedics and Related Research ${ }^{\circledR}$ editors and board members are on file with the publication and can be viewed on request. Clinical Orthopaedics and Related Research ${ }^{\mathbb{R}}$ neither advocates nor endorses the use of any treatment, drug, or device. Readers are encouraged to always seek additional information, including FDAapproval status, of any drug or device prior to clinical use.

Each author certifies that his or her institution approved or waived approval for the human protocol for this investigation and that all investigations were conducted in conformity with ethical principles of research.

J. C. Christensen, J. Brothers, G. J. Stoddard, M. B. Anderson,

C. E. Pelt, J. M. Gililland, C. L. Peters ( $\)$

Department of Orthopaedics, University of Utah, 590 S Wakara

Way, Salt Lake City, UT 84108, USA

e-mail: chris.peters@hsc.utah.edu

J. C. Christensen

Department of Physical Therapy, University of Utah,

Salt Lake City, UT, USA mechanics could provide improved physical function postoperatively and reduce the amount of residual symptoms. Advancements in the bicruciate-retaining (BCR) TKA implant design have been made, and based on these, we wished to compare the BCR with a more traditional cruciate-retaining (CR) implant.

Questions/purposes (1) Was there a difference in the risk of reoperation after primary TKA between BCR and CR implant designs? (2) Was there a difference in the radiographic findings of radiolucent lines (RLLs) between the implant designs? (3) Was there a difference in patient-reported and clinical outcomes between the two implant designs?

Methods Between January 2013 and May 2014, two surgeons performed 475 primary TKAs. During this time, 78 (16\%) of these were performed with BCR implants and 294 (62\%) with CR implants; the remainder were performed with anterior-stabilized or more constrained designs as a result of increased deformity and/or ligamentous deficiencies. During this period, the general indications for BCR TKA were arthritic knees with only slight to moderate deformity and sufficient ligamentous integrity of both the ACL and posterior cruciate ligament. The indications for CR TKA were similar other than these patients presented with a deficient ACL. A total of 66 (85\%) of the BCR and 237 $(81 \%)$ of the CR TKAs were available for followup at a minimum of 12 months or when reoperation occurred before 12 months (mean, 18 months; range, $2-32$ months). With the numbers available, there were no differences between the groups in terms of age and sex, but the patients undergoing CR TKA had a greater mean body mass index $(33 \pm 7$ versus $31 \pm 5 \mathrm{~kg} / \mathrm{m}^{2}, \mathrm{p}=0.032$ ). The frequency of early reoperation was compared between the groups as were radiographic evidence of RLL, patient-reported outcomes, and knee range of motion (ROM). 
Results Knees in the BCR group had a higher frequency of all-cause revision (5\% [three of 66] versus $1.3 \%$ [three of 237]; hazard ratio (HR), 7.44; $95 \%$ confidence interval [CI], 1.24-44.80; p = 0.028). Knees in the BCR group had a higher frequency of irrigation and débridement with component retention (HR, 0.07; 95\% CI, 0.02-0.28; p < $0.001)$. No differences were found between groups for subsequent manipulation (HR, 0.34; 95\% CI, 0.08-1.42; $\mathrm{p}=0.137)$. The proportion of RLLs was greater in the BCR group (HR, 2.93; 95\% CI, 1.62-5.32; p < 0.001) compared with the CR group. There were no differences between the groups in terms of the Physical Function Computerized Adaptive Test scores, Global10 scores or knee ROM outcomes.

Conclusions Preliminary short-term findings suggest the BCR implant has inferior survivorship and concerning radiographic findings when compared with a conventional CR implant with respect to complications after primary TKA. These findings raise concerns about the new BCR design; however, further randomized trials are necessary to determine superiority between alternative implant designs. Level of Evidence Level III, therapeutic study.

\section{Introduction}

As many as $25 \%$ of patients who undergo primary TKA report residual knee symptoms after surgery [2, 17]. As a result, alternative implant designs and surgical techniques have been proposed in an effort to improve patient-reported outcomes and clinical results after TKA [1]. Studies have shown that retention of the anterior cruciate ligament (ACL) results in more natural knee kinematics [9, 14, 25], thus potentially being a novel option for providing improved physical function postoperatively. Some speculate that bicruciate-retaining (BCR) TKA implants may produce superior knee mechanics and proprioception as a result of retention of the ACL and less trauma to the joint while potentially improving physical function postoperatively [21].

Conversely, as a result of an array of issues including a more challenging surgical technique, difficulty with ligament balancing, and debatable evidence of the true benefit of ACL retention, the BCR implant design has not seen wide use [6]. Further concerns have been raised with respect to tibial baseplate design [9], wear of polyethylene components [23], fracture of the bony tibial eminence [9], and ability to effectively correct knee deformity [6, 29]. Alterative implant designs such as the posterior cruciateretaining (CR) implant have established track records with regard to clinical efficacy, implant survivorship, and patient-reported outcomes [16]. Recently a new FDA-approved BCR implant was introduced based on an existing
TKA system with proven clinical effectiveness. We are unaware of any studies that have compared a series of BCR TKA implants with a conventional CR design with respect to clinical and radiographic outcomes during short-term postoperative recovery.

We therefore asked: (1) Was there a difference in the risk of reoperation after primary TKA between BCR and CR implant designs? (2) Was there a difference in the radiographic findings of radiolucent lines (RLLs) between the implant desings? (3) Was there a difference in patientreported and clinical outcomes between the two implant designs?

\section{Patients and Methods}

After receiving an exemption from the institutional review board, a retrospective comparative study of convenience sampling was conducted. Between January 2013 and May 2014, two surgeons (CLP, CEP) performed 475 primary TKAs (Fig. 1) that were identified by querying the enterprise data warehouse. During this time, 78 (16\%) of these were performed with BCR implants and 294 (62\%) with CR implants. The remainder was performed with

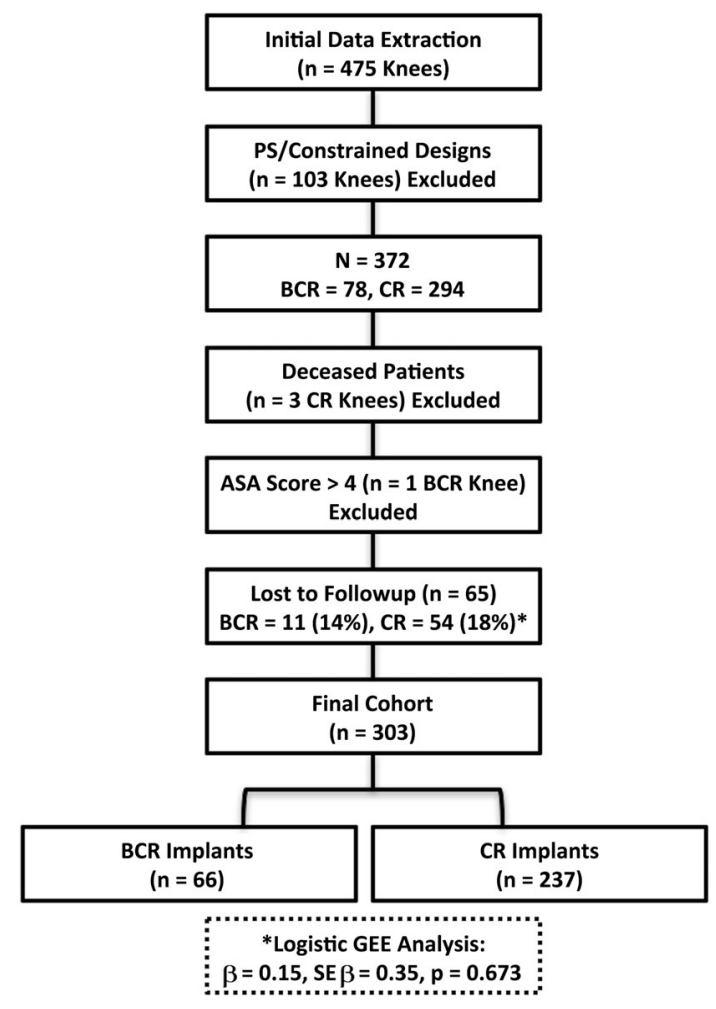

Fig. 1 This flowchart demonstrates the attrition of participants. Regarding the lost to followup (*), we found no difference in the proportion of patients lost between the BCR and CR implant groups $(\beta=0.15$, SE $\beta=0.35, p=0.673)$. 
anterior-stabilizing or more constrained designs. Indications for performing a more constrained design were based on the integrity of the posterior cruciate ligament (PCL), severe deformity, or ligamentous deficiencies as we have previously described [19]. Only the CR TKAs were used as the control group in an effort to minimize confounding variables. During this period, the general indications for BCR TKA were knees with bi- or tricompartmental arthritis with an intact ACL. In general, these resulted in knees with minimal coronal deformity or flexion contracture and the indications for CR TKA were if the integrity of the ACL was considered compromised based on clinical examination or intraoperative assessment. Of the initial 372 TKAs performed with BCR or CR implants, three patients (three CR knees) had died as a result of an unrelated health condition; one patient (one BCR knee) had an American Society of Anesthesiologists (ASA) physical status $>4$ and 65 patients (BCR, 11 [14\%]; CR, 54 [18\%], $\mathrm{p}=0.673$ ) did not have a minimum of 12-month followup or reoperation before that time (Fig. 1). Three hundred three knees at a minimum 12-month followup were included in the final analysis. There was no difference $(\mathrm{p}=0.673)$ in the proportion of patients lost to followup between groups with $14 \%$ (11 of 78) in the BCR knees and 18\% (54 of 294) in the CR knees. A total of 66 knees (65 patients [85\%]) of the BCR and 237 knees (232 patients [81\%]) of the CR TKAs were available for followup at a minimum of 12 months or when reoperation occurred before 12 months (mean, 18 months; range, 2-32 months). For simplicity, "knee" as opposed to patient was used as the unit of measure. Cement was used for both components in all of the BCR knees and for 162 of the 237 CR knees. Additionally, 71 of the CR knees had only the tibial component cemented and only one knee had only the femoral component cemented. The final three CR knees had cementless fixation for both the femoral and tibial components.

All data were collected from routine clinical care followup visits and extracted from the enterprise data warehouse or electronic medical record. The groups were not different with the numbers available in regard to age, gender, physical status classification, or knee osteoarthritis severity. However, patients receiving a CR implant had a higher mean body mass index (BMI) than did patients receiving a BCR implant ( $33 \pm 7$ versus $31 \pm 5 \mathrm{~kg} / \mathrm{m}^{2}, \mathrm{p}=$ 0.032; Table 1). Mean followup was slightly greater in the CR knees (1.52 years; range, 0.15-2.68 years) compared with the BCR knees (1.29 years; range, 0.42-2.26 years; $\mathrm{p}=0.002)$. The overall reoperation rate, excluding manipulations, was $5 \%$ (15 of 303). Most (nine of 15) of these reoperations were irrigation and débridements (I\&D) with retention of the femoral and tibial components. Twenty-two knees (7\%) underwent manipulation under anesthesia (MUA) as a result of postoperative arthrofibrosis (less than $90^{\circ}$ of flexion at 6 weeks postoperatively).

All TKAs were performed through a medial parapatellar approach with two FDA-approved implant designs (Zimmer Biomet, Warsaw, IN, USA). The goal for coronal alignment was $5^{\circ}$ valgus (anatomic axis) and $0^{\circ}$ mechanical axis. For CR knees, the tibia cut was neutral to the mechanical axis in the coronal plane and with $3^{\circ}$ to $5^{\circ}$ of posterior slope using an extramedullary guide. For BCR knees, the ACL was protected during femoral preparation and the tibia was prepared with a guide designed to preserve a bone island with the ACL insertion. The tibial cut was neutral to the mechanical axis and with $7^{\circ}$ of posterior slope in accordance with the manufacturer's recommendation. An initial medial release of the deep medial collateral ligament to the midsagittal line of the tibia was performed as part of the initial exposure. After bone preparation with measured resection bone cuts (posterior referencing), the symmetry of the flexion and extension gaps was investigated with trial components in place. Further medial, lateral, and PCL selective soft tissue release was performed to achieve balanced rectangular gaps. Once trial implants were placed, the ACL and PCL were evaluated for both competence and balance. All patients had the same postoperative regimen and followup schedule.

The 66 BCR TKAs included in this analysis were the first BCR knees implanted at our institution after the

Table 1. Baseline characteristics of study participants

\begin{tabular}{|c|c|c|c|c|}
\hline Characteristic & $\begin{array}{l}\text { Total knees } \\
(\mathrm{n}=303)\end{array}$ & $\begin{array}{l}\text { BCR group } \\
(\mathrm{n}=66)\end{array}$ & $\begin{array}{l}\text { CR group } \\
(\mathrm{n}=237)\end{array}$ & $\mathrm{p}$ value \\
\hline Age (years) & $64(9)$ & $65(7)$ & $63(10)$ & 0.259 \\
\hline Sex; number $(\%)$ male & $106(35 \%)$ & $25(38 \%)$ & $81(34 \%)$ & 0.564 \\
\hline BMI $\left(\mathrm{kg} / \mathrm{m}^{2}\right)$ & $32(7)$ & $31(5)$ & $33(7)$ & 0.032 \\
\hline ASA score, median (IQR) & $2(2-3)$ & $2(2-3)$ & $2(2-3)$ & 0.737 \\
\hline Kellgren-Lawrence OA grade, median (IQR) & $4(3-4)$ & $3.5(3-4)$ & $4(3-4)$ & 0.002 \\
\hline
\end{tabular}

Values represented as mean $(\mathrm{SD})$ unless otherwise stated; $\mathrm{BCR}=$ bicruciate-retaining; $\mathrm{CR}=$ cruciate-retaining; $\mathrm{BMI}=$ body mass index; $\mathrm{ASA}=$ American Society of Anesthesiologists; IQR = interquartile range; OA = osteoarthritis. 
implant received FDA approval. In this respect, the BCR results reflect an initial learning curve experience. One of the senior surgeons was involved with design of the implant (CLP) and the other was an early evaluator (CEP). Both orthopaedic surgeons who implanted the BCR knees had extensive prior cadaver work with the new BCR and routinely used the Vanguard CR (Zimmer Biomet) TKA system.

Querying of the enterprise data warehouse and chart reviews were performed by research staff with no direct involvement in the care of the patients. Reoperations included aseptic revision TKAs, I\&D with or without tibial polyethylene component exchange, and septic revision TKAs. We elected to review all causes for revision because anecdotally knowing the BCR TKAs required more surgical time (skin to skin). This was confirmed following the review where BCR TKAs had a mean surgical time of 104 minutes (range, 73-197 minutes) compared with CR TKAs with a mean 95 minutes (range, 60-188; $p=0.002$ ). Wound closure for both the BCR and CR implants was similar. For septic revisions, the presence of a periprosthetic joint infection was confirmed using the recommendations of the Musculoskeletal Infection Society (MSIS) [13, 18]. Separately, we reviewed the frequency of subsequent MUAs secondary to postoperative arthrofibrosis (less than $90^{\circ}$ of flexion at 6 weeks postoperatively). Although MUA for arthrofibrosis was not considered as a reoperation, it was included as a variable of interest for two reasons, the first being we sought to perform an all-encompassing review of the complications and the second was that we questioned whether a less evasive procedure would result in fewer cases of arthrofibrosis.

Component mechanical alignment, RLL, and/or fracture were evaluated postoperatively by a single orthopaedic surgeon (JB) according to the Knee Society Roentgenographic Evaluation System (KSRES) [8]. Radiographic imaging included standing AP, longstanding AP, lateral, and sunrise views. Records were reviewed for both surgical and nonsurgical complications. The following indices were recorded: presence of RLL, component migration, and subsidence. Radiolucent lines were assigned to Zones 1 to 7, as detailed in the KSRES, for the CR tibial components and Zones 1 to 6 were used for the BCR tibial baseplate as a result of the absence of a center keel (Fig. 2). Finally, tibial postoperative loosening or fracture was recorded. Nonprogressive RLLs are not normally a source of concern. However, because this study includes followup at short term, it is not possible to ascertain with certainty whether the RLL observed here will or will not be progressive, so it is difficult to know whether they will be of concern. In addition to comparing the presence of RLL between implant designs, KSRES scores were computed by adding the width $(\mathrm{mm})$ of the RLL for each zone on the tibial component in both the AP (Zones 1-7) and mediolateral (ML) (Zones 1-3) radiographic views (Fig. 2). The interpretation of the score has been published previously by Ewald [8] with a score of 10 or more signifying possible failure. Preoperatively, the severity of knee osteoarthritis was graded using the 0 to 4 scale as described by Kellgren and Lawrence [12].

Patient-reported outcomes were assessed using the National Institute of Health's Patient Reported Outcomes Measurement Information System (PROMIS) physical function computerized adaptive test (PF-CAT) and the Global Health short form (Global10). The PF-CAT is based on modern psychometric techniques using item response theory, which has been used in the educational field to optimize test administration for decades by reducing time constraints to the participant, test length, data entry errors, and organizational cost while preserving data efficacy [3, 4]. The PF-CAT includes a total of 124 physical function items across five categories related to activities of daily living and the Global10 is a 10 -item instrument representing five primary domains (physical functioning, pain, fatigue, emotional distress, social health) with cutoffs across domains $[4,5,10]$. Both outcome measures have demonstrated appropriate psychometric properties $[4,5$, 10, 11]. Preoperative physical health was assessed using the ASA Physical Status classification system, which is a 6point categorical scale and evaluates the degree of a patient's physical health before undergoing anesthesia and surgery [24].

Surgical knee ROM (degrees) was obtained by chart review and was measured at final followup by the clinicians by both physical examination and the use of a goniometer. It was used to quantify the maximum arc of knee mobility by expressing the full passive knee flexion and extension motion that could be obtained during clinical examination.

Baseline variables were compared between groups to detect potential confounding variables using a logistic generalized estimating equations (GEE) model with an unstructured covariance matrix to account for the correlation within patients who had bilateral knee involvement. Multivariable hazard ratios were estimated by a shared frailty Cox regression model with robust standard errors to account for the clustering of knees within patients. The underlying time metric was the date of reoperation minus date of index TKA for reoperations (revision TKA, I\&D procedures, and MUA). Both linear and logistic GEE regression models were used to examine differences between groups for postoperative RLL, PF-CAT, Global10, and knee ROM measures using an unstructured covariance matrix to account for the correlation within patients who had bilateral knee involvement. A subgroup analysis was also conducted using a similar model to evaluate differences between surgeons on the frequency of MUA after 


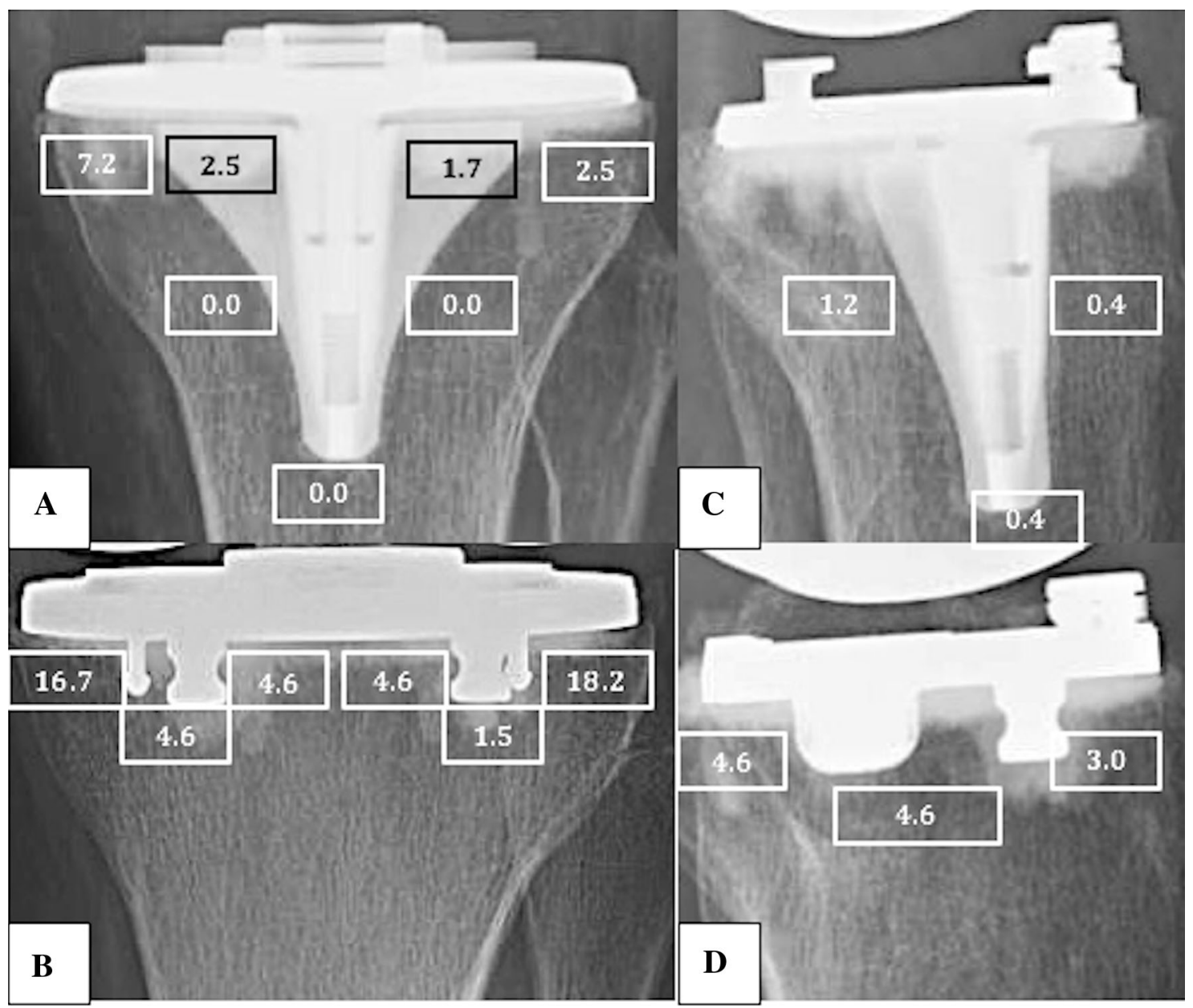

Fig. 2A-D This figure shows the location and proportion of radiolucent lines between implants on both $A P(C R=A, B C R=B)$ and mediolateral $(C R=C, B C R=D)$ views based on the Knee Society Total Knee Arthroplasty Roentgenographic Evaluation and Scoring System.

controlling for BMI. Missing data were imputed using the method of multiple multivariate imputation described by van Buuren et al. [27] as implemented in the STATA Version 14.1 software (College Station, TX, USA) [22].

Data representing the proportion of revision and isolated I\&D procedures were a concern for overfitting in the multivariable Cox regression models, where overfitting is an unreliable association produced by having too many predictor variables for the number of outcome events (Table 2). It has been shown that five events for each predictor in Cox regression is sufficient to avoid overfitting when the aim is to adjust for confounding [28], so given two predictors, group and BMI, we needed 10 events. For two outcomes, the number of events was less than 10, but we fitted the model controlling for BMI anyway. To determine if the large hazard ratios were reasonable, rather than a result of overfitting, we conducted a sensitivity analysis. Poisson regression of rates, using person-time denominators, also accounts for different times at risk or lengths of followup, but not as effectively as Cox regression. Both the rate ratio [15] and the hazard ratio [20] are estimates of relative risk. Exact Poisson regression is not subject to overfitting. To avoid lack of independence from having multiple surgeries for some patients, we randomly chose one of the two surgeries for those patients and combined this sample with those patients with one surgery. All outcome events remained in the sample. We then fit an exact Poisson regression model using group and BMI as the predictors. This model is known to be less statistically powerful than Cox regression. However, if the relative risks from exact Poisson regression remained large and approximately close to the Cox regression results, this would confirm the Cox regression results were not a product of overfitting. Because the relative risks from both statistical approaches are large and sufficiently similar, this confirms the Cox regression conclusions were not a product of overfitting. Data were analyzed using commercially available statistical software (Stata Version 14.1; Statacorp, LP, College Station, TX, USA). 
Table 2. Reoperation and complication rates*

\begin{tabular}{|c|c|c|c|c|}
\hline Variable & BCR group & CR group & $\begin{array}{l}\text { Adjusted HR* } \\
(95 \% \mathrm{CI})\end{array}$ & $\mathrm{p}$ value \\
\hline Reoperations, number $(\%)$ & $7(11)$ & $8(3)$ & $5.91(1.78-19.53)$ & 0.004 \\
\hline $\mathrm{I} \& \mathrm{D}$, number $(\%)$ & $5(8)$ & $4(2)$ & $0.07(0.02-0.28)$ & $<0.001$ \\
\hline Isolated & $3(5)$ & $1(<1)$ & $12.9(1.34-123.6)$ & 0.027 \\
\hline With polyethylene exchange & $2(3)$ & $3(1)$ & $3.07(0.53-18.94)$ & 0.207 \\
\hline Revision TKA, number (\%) & $3(5)$ & $3(1)$ & $7.44(1.24-44.80)$ & 0.028 \\
\hline Septic & $1(2)$ & $1(<1)$ & $6.6(0.75-58.3)$ & 0.089 \\
\hline Aseptic & $2(3)$ & $2(1)$ & $8.41(0.83-84.9)$ & 0.071 \\
\hline Femoral loosening & 0 & 2 & & \\
\hline ACL impingement & 1 & 0 & & \\
\hline Metal allergy/tibial loosening & 1 & 0 & & \\
\hline Manipulations, number (\%) & $2(3)$ & $20(8)$ & $0.34(0.08-1.42)$ & 0.137 \\
\hline Radiolucent lines, number (\%) & $20(30)$ & $36(15)$ & $2.93(1.62-5.32)$ & $<0.001$ \\
\hline
\end{tabular}

*Each row of the table reports that hazard ratio and $\mathrm{p}$ value from a separate multivariable shared frailty Cox regression while controlling for body mass index; $\mathrm{BCR}=$ bicruciate-retaining; $\mathrm{CR}=$ cruciate-retaining; $\mathrm{HR}=$ hazard ratio; $\mathrm{CI}=$ confidence interval; $\mathrm{I} \& \mathrm{D}=$ irrigation and débridement; $\mathrm{ACL}=$ anterior cruciate ligament.

\section{Results}

Knees in the BCR group had a higher frequency of reoperation of all-cause revision (hazard ratio [HR], 7.44; 95\% confidence interval $[\mathrm{CI}], 1.24-44.8 ; \mathrm{p}=0.028$; Table 2). The one BCR septic revision only had an elevated erythrocyte sedimentation rate and C-reactive protein and did not end up meeting the MSIS criteria for periprosthetic joint infection [18] knowing there was no evidence of overt infection on revision and intraoperative cultures were negative. Of note, the femoral revisions in the CR group were both for aseptic loosening of cementless femoral components. The two tibial revisions in the BCR group were for ACL impingement in one case and aseptic tibial loosening with suspected metal allergy in the other. Knees in the BCR group had a higher frequency of I\&D with component retention (HR, 0.07; 95\% CI, 0.02-0.28; p < 0.001; Table 2). Of these nine I\&Ds, five in the BCR group were for hematoma with drainage (two), possible infection (two), and wound dehiscence (one). The other four in the CR group were resulting from hematoma (two), possible infection (one), and residual knee pain (one). A BCR knee with isolated I\&D had a subsequent I\&D with polyethylene exchange and one of the BCR knees that underwent initial I\&D with polyethylene exchange went onto revision TKA. With the numbers available, we found no differences between groups for MUA (HR, 0.34; 95\% CI, 0.08-1.42; $\mathrm{p}=0.137)$. Of the MUAs, two (3\%) were in the BCR group and $20(8 \%)$ were in the CR group. A subanalysis comparing the frequency of MUA between surgeons after controlling for BMI also revealed no differences with the numbers available (HR, 1.10; 95\% CI, 0.48-2.50; $\mathrm{p}=0.825)$.
The proportion of RLL was greater (HR, 2.93; 95\% CI, $1.62-5.32 ; \mathrm{p}<0.001$ ) in the BCR group 30\% (20 of 66) compared with the CR group 15\% (36 of 237). Knees in the BCR group demonstrated higher KSRES scores of RLL in both the AP $(\beta=0.37, \mathrm{z}$-score $=4.10, \mathrm{p}<0.001)$ and mediolateral $(\beta=0.10, \mathrm{z}$-score $=2.70, \mathrm{p}=0.007)$ radiographs compared with the CR group.

There were no postoperative differences between groups regarding physical function when comparing the PROMIS PF-CAT T-scores with a mean difference of 1 unit $(95 \%$ $\mathrm{CI},-3$ to 1 ). The BCR group had a mean (SD) PF CAT T-score of 43 (8) compared with a mean score of 44 (8) in the $\mathrm{CR}$ group $(\mathrm{p}=0.338)$. There was no difference in PROMIS Global 10 T-scores. Finally, the mean (SD) postoperative knee ROM in the BCR group was 122 (8) and the CR group had a mean (SD) of $120(10)(p=0.364$; Table 3$)$.

\section{Discussion}

It is estimated that as many as $25 \%$ of patients who undergo primary TKA report residual knee symptoms after surgery $[2,17]$. One possible reason for the high level of patients experiencing residual symptoms is potentially that conventional TKA, with sacrifice of the ACL, poorly replicates normal knee kinematics and proprioception. Although biomechanical studies have concluded that preservation of the ACL results in more normal joint mechanics, we are unaware of any in vivo studies investigating pertinent clinical outcomes that compare a BCR design with an alternative implant [1, 6]. Bicruciate-retaining TKA has been proposed as a possible solution and 
Table 3. Clinical outcome measures

\begin{tabular}{llll}
\hline Variable & $\begin{array}{l}\text { BCR group } \\
(\mathrm{n}=66)\end{array}$ & $\begin{array}{l}\text { CR group } \\
(\mathrm{n}=237)\end{array}$ & p value \\
\hline PF-CAT & $43(8)$ & $44(8)$ & 0.338 \\
Global Health & $4(1)$ & $3(1)$ & 0.482 \\
Global Pain & $3(2)$ & $4(2)$ & 0.384 \\
Global Mental & $50(9)$ & $50(8)$ & 0.959 \\
Global Social & $4(1)$ & $4(1)$ & 0.968 \\
Arc of Motion & $122(8)$ & $120(10)$ & 0.364 \\
\hline
\end{tabular}

Values represented as mean $(\mathrm{SD})$ unless otherwise stated; $\mathrm{BCR}=$ bicruciate-retaining; $\mathrm{CR}=$ cruciate-retaining; $\mathrm{PF}-\mathrm{CAT}=$ physical function computerized adaptive test.

in the past has shown clinical promise [7]. Our results demonstrated a higher rate of early reoperation, revision, and RLL in the BCR TKA group compared with the CR TKA group. There was no difference in patient-reported clinical outcomes between the groups.

The study had several limitations as described subsequently. First, the BCR group represented the first 66 BCR TKAs performed at our institution. Although the operating surgeons were experienced TKA surgeons, this represented the initial learning curve with a new implant design with ACL retention. The surgical technique of TKA with ACL retention is appreciably more difficult than conventional TKA. This, together with the learning curve for ligament balancing and implant fixation, may partially explain the higher reoperation and RLL in the BCR group. This may be particularly relevant given the relatively high rate of reoperations in the BCR group, which may be indicative of increased surgical technical difficulty. Second, our CR cohort had a longer followup duration then the BCR, which could have introduced possible bias affecting the results. Third, we had between-group discrepancies in BMI, which potentially could suggest selection bias favoring the BCR group. However, based on our findings indicating the opposite effect, we suspect that this source of error is unlikely. Fourth, the indications for retention of the ACL were relative and left to the discretion of the operating surgeon. Related to this was the fact that the study was a retrospective cohort design comparing two nonrandomized groups of patients based on type of treatment. A multicenter randomized study comparing similar groups is currently underway and should provide a more rigorous analysis of the BCR TKA group.

Reoperation was more frequent in the BCR group and most of these were for wound-related complications. However, the overall risk of revision was also higher in the BCR group ( $5 \%$ versus $1 \%$ ). In the longest reported followup study on BCR knees, Sabouret et al. [23] reported a survival rate of $82 \%$ with revision surgery as the endpoint.
The reasons for revision included $12 \%$ for polyethylene wear and $4 \%$ for aseptic component loosening. Similarly, Townley [26] reviewed the 11-year outcome of 532 anatomic TKAs. The outcomes were $89 \%$ good to excellent results and $4 \%$ poor or failed. The implant failures included 10 with loose tibial implants. Neither Sabouret et al. [24] nor Townley $[23,26]$ commented on the early complication or reoperation nor the potential for a learning curve effect early in the operative experience.

In terms of RLL, the BCR group had a higher frequency of RLL than the CR group. In addition, the distribution of RLL was different between the two groups with the BCR group having a high percentage of RLL at the periphery (medial and lateral) of the tibial implant. This finding is likely related to the fact that tibial preparation in BCR knees is more difficult as a result of an inability to fully subluxate the tibia anteriorly as a result of the presence of the ACL. This may result in inaccurate tibial resection and poorer visualization during cementation. Prior reports of predicate BCR designs have not analyzed in detail the presence or absence of RLL and their potential correlation with tibial loosening. The clinical ramifications of these early RLL findings are not yet known. It is widely accepted that nonprogressive RLLs are not normally a source of concern without presence of loosening, but because the followup in this study was short and it is not possible to know whether these RLLs will progress to implant loosening, we felt it important to report them.

Our results failed to demonstrate any differences in patient-reported outcomes between the two groups using the PROMIS PF-CAT and Global10 instruments. Older studies on BCR TKA have primarily focused on implant survivorship and surgeon-reported outcomes [7, 23, 26] rather than on patient-reported outcomes, and so comparisons are difficult to make. Pritchett [21] demonstrated strong patient preference for a BCR $(89 \%)$ compared with other TKA designs in patients who underwent staged bilateral TKA.

Compared with conventional CR TKA, a new BCR TKA demonstrated an increased frequency of early reoperation and a greater frequency of RLL. There were no differences in patient-reported clinical outcomes between the two designs. These results could be explained by early learning curve experiences or truly inferior outcomes resulting from the $\mathrm{BCR}$ design. Using this data set and early clinical findings, we are unable to demonstrate any advantages of the new device with the measured outcomes. Given the costs and risks associated with novel implants, readers should be cautioned about widespread adoption in clinical practices until properly designed studies show not only equivalence, but ultimately superiority. Future welldesigned randomized controlled trials will be necessary to support the use of new technologies, including this implant 
design. With this in mind, we are participating in a multicenter randomized clinical trial comparing the two implants for superiority in reoperation, radiographic findings, and clinical outcomes.

Acknowledgments We thank David Evans and Nadia Hamad for their contributions to this study.

\section{References}

1. Andriacchi TP. Functional analysis of pre and post-knee surgery: total knee arthroplasty and ACL reconstruction. J Biomech Eng. 1993;115:575-581.

2. Bourne RB, Chesworth BM, Davis AM, Mahomed NN, Charron KD. Patient satisfaction after total knee arthroplasty: who is satisfied and who is not? Clin Orthop Relat Res. 2010;468:57-63.

3. Broderick JE, DeWitt EM, Rothrock N, Crane PK, Forrest CB. Advances in Patient-Reported Outcomes: The NIH PROMIS ${ }^{\circledR}$ Measures. EGEMS (Wash DC). 2013;1:1015.

4. Broderick JE, Schneider S, Junghaenel DU, Schwartz JE, Stone AA. Validity and reliability of patient-reported outcomes measurement information system instruments in osteoarthritis. Arthritis Care Res. 2013;65:1625-1633.

5. Cella D, Riley W, Stone A, Rothrock N, Reeve B, Yount S, Amtmann D, Bode R, Buysse D, Choi S, Cook K, Devellis R, DeWalt D, Fries JF, Gershon R, Hahn EA, Lai JS, Pilkonis P, Revicki D, Rose M, Weinfurt K, Hays R. The Patient-Reported Outcomes Measurement Information System (PROMIS) developed and tested its first wave of adult self-reported health outcome item banks: 2005-2008. J Clin Epidemiol. 2010;63: 1179-1194.

6. Cherian JJ, Kapadia BH, Banerjee S, Jauregui JJ, Harwin SF, Mont MA. Bicruciate-retaining total knee arthroplasty: a review. J Knee Surg. 2014;27:199-205.

7. Cloutier JM, Sabouret P, Deghrar A. Total knee arthroplasty with retention of both cruciate ligaments. A nine to eleven-year follow-up study. J Bone Joint Surg Am. 1999;81:697-702.

8. Ewald FC. The Knee Society total knee arthroplasty roentgenographic evaluation and scoring system. Clin Orthop Relat Res. 1989;248:9-12.

9. Halewood C, Traynor A, Bellemans J, Victor J, Amis AA. Anteroposterior laxity after bicruciate-retaining total knee arthroplasty is closer to the native knee than ACL-resecting TKA: a biomechanical cadaver study. J Arthroplasty. 2015;30:23152319.

10. Hays RD, Bjorner JB, Revicki DA, Spritzer KL, Cella D. Development of physical and mental health summary scores from the patient-reported outcomes measurement information system (PROMIS) global items. Qual Life Res. 2009;18:873-880.

11. Hung M, Clegg DO, Greene T, Saltzman CL. Evaluation of the PROMIS physical function item bank in orthopaedic patients. J Orthop Res. 2011;29:947-953.
12. Kellgren JH, Lawrence JS. Radiological assessment of osteoarthrosis. Ann Rheum Dis. 1957;16:494-502.

13. Koh IJ, Cho WS, Choi NY, Parvizi J, Kim TK. How accurate are orthopedic surgeons in diagnosing periprosthetic joint infection after total knee arthroplasty? A multicenter study. Knee. 2015;22:180-185.

14. Komistek RD, Allain J, Anderson DT, Dennis DA, Goutallier D. In vivo kinematics for subjects with and without an anterior cruciate ligament. Clin Orthop Relat Res. 2002;404:315-325.

15. Last J. A Dictionary of Epidemiology. 3rd ed. New York, NY, USA: Oxford University Press; 1995:145.

16. Most E, Zayontz S, Li G, Otterberg E, Sabbag K, Rubash HE. Femoral rollback after cruciate-retaining and stabilizing total knee arthroplasty. Clin Orthop Relat Res. 2003;410:101-113.

17. Noble PC, Conditt MA, Cook KF, Mathis KB. The John Insall Award: Patient expectations affect satisfaction with total knee arthroplasty. Clin Orthop Relat Res. 2006;452:35-43.

18. Parvizi J, Zmistowski B, Berbari EF, Bauer TW, Springer BD, Della Valle CJ, Garvin KL, Mont MA, Wongworawat MD, Zalavras CG. New definition for periprosthetic joint infection: from the Workgroup of the Musculoskeletal Infection Society. Clin Orthop Relat Res. 2011;469:2992-2994.

19. Peters CL, Mulkey P, Erickson J, Anderson MB, Pelt CE. Comparison of total knee arthroplasty with highly congruent anterior-stabilized bearings versus a cruciate-retaining design. Clin Orthop Relat Res. 2014;472:175-180.

20. Pocock SJ, Clayton TC, Altman DG. Survival plots of time-toevent outcomes in clinical trials: good practice and pitfalls. Lancet. 2002;359:1686-1689.

21. Pritchett JW. Patients prefer a bicruciate-retaining or the medial pivot total knee prosthesis. J Arthroplasty. 2011;26:224-228.

22. Royston P, Sauerbrei W. A new measure of prognostic separation in survival data. Stat Med. 2004;23:723-748.

23. Sabouret P, Lavoie F, Cloutier JM. Total knee replacement with retention of both cruciate ligaments: a 22-year follow-up study. Bone Joint J. 2013;95:917-922.

24. Saklad M. Grading of patients for surgical procedures. Anesthesiology. 1941;2:281-284.

25. Stiehl JB, Komistek RD, Cloutier JM, Dennis DA. The cruciate ligaments in total knee arthroplasty: a kinematic analysis of 2 total knee arthroplasties. J Arthroplasty. 2000;15:545-550.

26. Townley CO. The anatomic total knee resurfacing arthroplasty. Clin Orthop Relat Res. 1985;192:82-96.

27. van Buuren S, Boshuizen HC, Knook DL. Multiple imputation of missing blood pressure covariates in survival analysis. Stat Med. 1999;18:681-694.

28. Vittinghoff E, McCulloch CE. Relaxing the rule of ten events per variable in logistic and Cox regression. Am J Epidemiol. 2007; 165:710-718.

29. Zumbrunn T, Varadarajan KM, Rubash HE, Malchau H, Li G, Muratoglu OK. Regaining native knee kinematics following joint arthroplasty: a novel biomimetic design with ACL and PCL preservation. J Arthroplasty. 2015;30:2143-2148. 